\title{
Atividade acaricida de óleos essenciais sobre Tetranychus ludeni (Zacher) (Acari: Tetranychidae) em duas cultivares de algodoeiro
}

\author{
Yasmin Bruna de Siqueira BEZERRA ${ }^{1 *}$, José Vargas de OLIVEIRA ${ }^{1}$, Taciana Keila dos Anjos RAMALHO ${ }^{1}$, \\ Douglas Rafael e Silva BARBOSA ${ }^{1}$, Carlos Romero Ferreira de OLIVEIRA², \\ Cláudia Helena Cysneiros Matos de OLIVEIRA ${ }^{1}$, Ibsen Francisco Assis LIMA NETO ${ }^{1}$
}

\author{
${ }^{1}$ Universidade Federal Rural do Pernambuco, Recife, PE, Brasil. \\ ${ }^{2}$ Instituto Federal de Educação, Ciência e Tecnologia do Maranhão, Bacabal, MA, Brasil. \\ E-mail: yasmin.bbezerra25@gmail.com
}

\begin{abstract}
Recebido em setembro/2018; Aceito em março/2019.
RESUMO: O presente trabalho teve como objetivo avaliar os efeitos repelentes e as taxas de crescimento populacional de óleos essenciais de Corymbia citriodora, Ocimum basilicum e Myracroduon urundeuva sobre o ácaro vermelho Tetranychus ludeni (Zacher) em algodoeiro de fibra colorida. Para o teste de repelência, óleos de Corymbia citriodora, Ocimum basilicum e Myracroduon urundeuva foram testados em diferentes concentrações sobre fêmeas adultas de $T$. ludeni, assim como para o teste de crescimento populacional, onde as cultivares foram tratadas com óleos essenciais. O efeito repelente foi verificado para todos os óleos testados nas duas cultivares de algodão colorida. As taxas instantâneas de crescimento populacional para T. ludeni nas cultivares de algodoeiro BRS Verde e BRS Rubi foram todas positivas $\left(r_{i}>0\right)$, indicando que a população está em estado ascendente, no entanto, a população cresceu a uma taxa menor quando comparada ao controle. Os óleos essenciais de $C$. citriodora, $O$. basilicum e $M$. urundeuva apresentam efeito acaricida e potencial controle alternativo de $T$. ludeni em algodoeiro de fibras coloridas sem causar danos ao algodoeiro.
\end{abstract}

Palavras-chave: ácaro vermelho; algodão colorido; controle alternativo; taxa instantânea de crescimento; repelência.

\section{Acaricidal activity of essential oils on red mite Tetranychus ludeni (Zacher) (Acari: Tetranychidae) in two cotton cultivars}

\begin{abstract}
The objective of this work was to evaluate the repellent and population growth rates of essential oils of Corymbia citriodora, Ocimum basilicum and Myracroduon urundeuva on the red mite Tetranychus ludeni (Zacher) in cotton fiber. For the repellency test, oils of Corymbia citriodora, Ocimum basilicum and Myracroduon urundeuva were tested in different concentrations on adult females of T. ludeni, as well as for the population growth test, where the cultivars were treated with essential oils. The repellent effect was verified for all tested oils the two cultivars of colored cotton. The instant population growth rates for T. ludeni in the cultivars BRS Verde and BRS Rubi were all positive ( $\mathrm{ri}>0$ ), indicating that the population is in an upward, however, the population grew at a lower rate when compared to the control. The essential oils of C. citriodora, O. basilicum and $M$. urundeuva have an acaricidal effect and potential alternative control of T. ludeni in cotton from colored fibers without causing damage to the cotton.
\end{abstract}

Keywords: Red mite; colored cotton; essential oils; instant growth rate; repellency.

\section{INTRODUÇÃO}

O algodoeiro, Gossypium hirsutum Linnaeus é uma das culturas mais importantes do mundo, sendo cultivado em mais de 80 países e gera movimentação financeira mundial estimada em 30 bilhões de dólares (ANUÁRIO BRASILEIRO DO ALGODÃO, 2018). O seu cultivo é muito importante para a economia nordestina, gerando milhares de empregos e abastecendo a indústria têxtil desta região, e com consumo anual de cerca 1,9 milhão de toneladas de pluma, algo em torno de $27 \%$ a mais que a safra anterior (CONAB, 2018). A cotonicultura está entre as atividades agrícolas com maior custo de produção.

No Brasil o gasto com insumos agrícolas representa cerca de $55 \%$ do total da produção, sendo que $25 \%$ deste total é destinado exclusivamente na compra de inseticidas (SANTOS, 2013). O algodão colorido e derivados representam, para alguns segmentos da indústria de têxteis e confecções do Estado da Paraíba, uma alternativa de inserção competitiva nos mercados, dos quais a produção do algodão branco e derivados foi alijada (BATISTA, 2008). Assim, os algodões de fibras coloridas (BRS Rubi e BRS Verde), entre outras, tornaram-se importantes na economia, uma vez que essas cultivares representam o crescimento deste mercado e a perspectiva de um negócio duradouro, especialmente por ser um produto diferenciado e, portanto, de maior valor agregado (EMBRAPA ALGODÃO, 2006).

Pragas podem limitar a produção de algodão. Dentre os artrópodes pragas, ácaros (Acari: Tetranychidae) estão entre as pragas mais importantes do mundo, com perdas de safra e custos de controle de milhões de dólares (ADANGO et al., 2006). O ácaro vermelho, Tetranychus ludeni Zacher (Acari: Tetranychidae), é altamente polífago e ocorre no campo 
durante quase todo o ano (KAIMAL; RAMANI, 2011). T. ludeni é uma espécie cosmopolita que ocorre em diversas plantas hospedeiras, constituindo uma das suas principais espécies-praga. É amplamente encontrado nos trópicos, desde o sul dos Estados Unidos, México, Américas Central e do Sul até a Austrália (ZHANG, 2002). Durante o processo de alimentação introduz os estiletes nos tecidos das plantas, suga o conteúdo celular extravasado, injeta toxinas e reguladores de crescimento (FLECHTMANN, 1985). Assim, dependendo da infestação, os ácaros podem contribuir na perda de $30 \%$ na produção de algodão em caroço e de $14,8 \%$ nas características das fibras (GALLO et al., 2002).

A principal forma de controle do T. ludeni na cultura do algodão, atualmente, é o uso de acaricidas, no entanto, mesmo quando são realizadas pulverizações regulares, existem muitos casos em que o controle se torna ineficiente. O uso intenso de produtos químicos sintéticos tem exposto muitas populações de ácaros-praga a uma intensa pressão de seleção e, consequentemente, ao desenvolvimento de indivíduos resistentes e aumento da população de pragas (KOUSIK et al. 2007; KUMRAL et al. 2010; MOSCARDINI et al. 2014).

Visando estudar táticas alternativas ao controle químico para o manejo de pragas, os inseticidas botânicos ou plantas inseticidas, surgem como uma tática promissora, que permitirá a pouca ou nenhuma presença de resíduos agrotóxicos em alimentos. Enquadram-se nessa categoria, os extratos e óleos essenciais obtidos de plantas medicinais e aromáticas (GOMES et al., 2016). Muitos compostos de origem vegetal têm sido isolados, como os terpenóides, limonóides, alcalóides e acetogeninas, apresentando propriedades inseticidas. Provocam mortalidade, repelência, deterrência na alimentação e oviposição e afetam o crescimento dos insetos (ROSADOAGUILAR et al., 2017). Os inseticidas botânicos devem ser utilizados como um método de controle eficaz e que sejam ecologicamente seguros e com redução dos custos com sintéticos, tornando-se prática adequada à agricultura sustentável (LUPI et al., 2013; KHATER et al., 2016).

A aroeira-do-sertão (Myracrodruon urundeuva Allemão) é uma espécie arbórea pertencente da família Anacardiaceae, cuja distribuição natural limita-se à América do Sul, sendo nativa do Brasil e amplamente distribuída nas regiões Nordeste, Sudeste e Centro Oeste (CECÍLIO et al., 2015). Tem alto valor terapêutico, o qual foi relatado por De Mendonça Albuquerque et al. (2011), em razão de que na casca encontram-se vários componentes fitoquímicos que possuem propriedades anti-inflamatórias. Também foram estudadas atividades inseticidas, antimicrobianas e cicatrizante, demonstrando que essas atividades foram atribuídas à presença de compostos bioativos como taninos e flavonoides (NAPOLEÃO et al., 2012; GOMES et al., 2013).

O eucalyptus é uma árvore da família Myrtaceae, e tem sua origem na Austrália, Nova Guiné, Indonésia e Timor (ARAÚJO-FILHO et al., 2018). Entre as espécies de eucalipto utilizado para a extração de óleos essenciais, Corymbia citriodora (Hook.) K.D. Hill \& L.A.S. Johnson (antigo Eucalyptus citriodora Hook.), é o mais utilizado. O óleo essencial de $C$. citriodora é extraído de folhas secas, e tem como principal constituinte o monoterpenóide citronelal (60$80 \%$ ) (HASEGAWA et al., 2008). Diversos trabalhos demonstraram bioatividades do óleo de $C$. citriodora como atividades antioxidantes (SINGH et al., 2012), antifúngico (TOLBA et al., 2015), antibacteriano, inseticida como repelente de insetos que se alimentam de suas folhas
(HUSSEIN et al., 2017), e até mesmo acaricida (CLEMENTE et al., 2010).

O gênero Ocimum L. pertence à família Lamiaceae, é cosmopolita e cultivada para fins medicinais e culinários, compreende aproximadamente 65 espécies que se distribuem amplamente no mundo, sobretudo nas regiões tropicais e subtropicais (KHOURY et al., 2016).

A espécie Ocimum gratissimum L., conhecida popularmente como alfavaca, alfavacão ou alfavaca-cravo é um subarbusto aromático, com até um metro de altura, originário do Oriente e subespontâneo em todo território brasileiro. Seu óleo essencial é rico em eugenol, terpenóides e flavonóides (MAMADALIEVA et al., 2017) e estudos têm comprovado sua eficácia contra diversos microrganismos e atividades inseticidas (NASCIMENTO et al., 2015).

Uma técnica alternativa para avaliar a toxicidade de inseticidas e acaricidas tem sido a estimativa da taxa instantânea de crescimento $\left(\mathrm{r}_{\mathrm{i}}\right)$, que permite avaliar os efeitos letais e subletais desses produtos sobre uma população, após um tempo previamente determinado (STARK; BANKS, 2003). Essa técnica foi avaliada, em diferentes pragas, como por exemplo, Aphis gossyii (ANDRADE et al., 2012), Myzus persicae e Eriopis connexa (VENZON et al., 2007).

Considerando a importância da cultura do algodoeiro e a necessidade do estabelecimento de formas de controle alternativas, objetivou-se neste trabalho avaliar o efeito de óleos essenciais no crescimento populacional e na repelência de fêmeas de T. ludeni, em cultivares de fibras coloridas.

\section{MATERIAL E MÉTODOS}

A pesquisa foi desenvolvida no período de julho de 2012 a novembro de 2013, no Laboratório de Entomologia/Ecologia da Unidade Acadêmica de Serra Talhada (UAST), Universidade Federal Rural de Pernambuco (UFRPE).

\subsection{Cultivares de algodoeiro utilizadas.}

Foram testadas as cultivares de fibras coloridas (BRS Verde e BRS Rubi) desenvolvidos pela Embrapa Algodão, localizada em Campina Grande, Paraíba, Brasil. Em todos os experimentos foram utilizadas folhas com 25 dias de idade, as quais foram lavadas com água destilada, mantidas sobre papel toalha para a retirada do excesso de umidade e posteriormente utilizadas nos experimentes de laboratório.

\subsection{Criação de Tetranychus ludeni}

Os ácaros foram criados, em laboratório, em arenas de plástico com $17 \mathrm{~cm}$ de diâmetro e dois centímetros de altura, contendo folhas de $C$. ensiformes com a face abaxial voltada para cima, sobrepostas a discos de espuma de polietileno e papel filtro. A margem da folha foi contornada com algodão umedecido em água destilada para evitar a fuga dos ácaros. A folha foi trocada semanalmente. As arenas foram mantidas em câmara climática do tipo B.O.D. à temperatura de $25 \pm 2^{\circ} \mathrm{C}, 70$ $\pm 10 \%$ UR e 12 horas de fotofase (adaptada de Ferreira et al. 2006) e em mudas de algodoeiro, nas plantas as criações foram mantidas em gaiolas de madeira $\left(1,0 \mathrm{~m}^{2}\right)$ revestidas com organza, sobre plantas de feijão de porco Canavalia ensiformes (L.) DC. (Fabaceae). Logo após a abertura completa do primeiro par de folhas cotiledonares, fez-se a infestação, com ácaros provenientes da criação estoque do laboratório. Semanalmente, fez-se infestações em novas plantas, colocando-se folhas com colônias do ácaro em contato com folhas sadias. 


\subsection{Obtenção dos óleos essenciais}

Folhas de $M$. urundeuva foram coletadas em área da Caatinga situada nos arredores da UAST da UFRPE. A exsicata desta planta foi depositada sob o número \#455 no Herbário do Semiárido Brasileiro (HESBRA) da UFRPE/UAST. O óleo essencial foi obtido a partir de folhas frescas, as quais foram trituradas separadamente com água destilada e o óleo obtido por meio da técnica de hidrodestilação por $2 \mathrm{~h}$ em aparelho tipo Clevenger modificado. Após isso as frações obtidas foram separadas da água por diclorometano e secas com sulfato de sódio anidro $\left(\mathrm{Na}_{2} \mathrm{SO}_{4}\right)$ e levadas posteriormente ao rota-evaporador, onde foi retirado o diclorometano e obtido apenas o óleo essencial. Os óleos foram armazenados sob refrigeração $\left(+5^{\circ} \mathrm{C}\right)$ em recipientes de vidros escuros vedados.

\subsection{Preparação das Caldas dos Óleos Essenciais}

Os óleos essenciais foram diluídos em água destilada e em dimetil sulfóxido $2 \%$ (DMSO) e preparados nas concentrações de 0,$5 ; 0,8 ; 1,25$ e $1,5 \%$. As concentrações foram estimadas, por meio de testes preliminares, obtendo-se mortalidade em torno de 5 e $95 \%$, para o estabelecimento das outras concentrações, por extrapolação, utilizando-se a seguinte fórmula (FINNEY et al., 1971).

$$
\mathrm{Q}=\mathrm{n}+1\left(\mathrm{a}_{\mathrm{n}} / \mathrm{a}_{1}\right)^{0,5}
$$

em que: $q$ = razão da progressão geométrica (p.g.); $n$ = número de concentrações a extrapolar; $a_{n}=$ limite superior da p.g. (concentração que provocou mortalidade de cerca de $95 \%$, determinada por meio de teste preliminar); $a_{1}=$ limite inferior da p.g. (concentração que provocou mortalidade de cerca de $5 \%$, determinada por meio de teste preliminar).

\subsection{Atividade repelente dos óleos essenciais sobre T. ludeni em} cultivares de algodoeiro

Foram utilizados discos de folhas de $3,5 \mathrm{~cm}$ de diâmetro para cada cultivar de algodão com a idade de 25 dias após o plantio. Os experimentos foram efetuados em arenas contendo dois discos de folha de cada cultivar de algodão (BRS Rubi e BRS Verde) de 3,5cm de diâmetro obtidos de folhas da parte mediana das plantas, sendo um disco tratado com cada óleo essencial na concentração de $1,5 \%$ (tratamento) e o outro com água destilada (testemunha). Um disco foi imerso durante cinco segundos na calda dos óleos essenciais e o outro disco foi imerso também por cinco segundos na testemunha e, posteriormente, secos por 30 minutos em temperatura ambiente; em seguida, foram colocados em placas de Petri plásticas, contendo ágar-água a $1 \%$. Os discos foram interligados por uma lamínula $(18$ x $18 \mathrm{~mm})$, na qual foram liberadas 10 fêmeas adultas do ácaro (Adaptado por Esteves Filho et al. 2010). Cada óleo essencial foi testado, individualmente, empregando-se o teste $\mathrm{t}$ pareado com escolha, constando de dois tratamentos (óleo essencial versus testemunha) e cinco repetições.

Os bioensaios foram avaliados após $48 \mathrm{~h}$, observando-se o número de ácaros vivos em cada disco. Para o cálculo do índice de repelência (IR\%) utilizou-se a fórmula.

$$
\mathrm{IR}=2 \mathrm{G} /(\mathrm{G}+\mathrm{P}) \quad \text { (equação 2) }
$$

em que: $\mathrm{G}=\%$ de ácaros atraídos no tratamento; $\mathrm{P}=\%$ de ácaros atraídos na testemunha. Os valores de IR variam entre zero e dois, sendo que IR = 1 indica repelência semelhante entre o tratamento e a testemunha (tratamento neutro), IR > 1 indica menor repelência do tratamento em relação à testemunha (tratamento atraente) e IR $<1$ corresponde à maior repelência do tratamento em relação à testemunha (tratamento repelente). Os resultados foram submetidos ao teste t para dados pareados e analisados, mediante o programa computacional SAS version 8.02 (SAS INSTITUTE, 2001).

2.6 Taxa instantânea de crescimento populacional de Tetranychus ludeni em cultivares de algodoeiro tratadas com óleos essenciais

Foram utilizados discos de folha de $5,0 \mathrm{~cm}$ de diâmetro das cultivares de algodoeiro coloridas (BRS Rubi e BRS Verde) após 25 dias e idade, cultivadas no Campus da UAST. Os discos foram imersos durante cinco segundos nas caldas dos óleos de $C$. citriodora, O. gratissimum e $M$. urundeuva constando de quatro concentrações $(0,5,0,8,1,25$ e $1,5 \%)$ e em água destilada (testemunha), e secos por 30 minutos à temperatura ambiente. Em seguida, foram colocados em placas de Petri plásticas, contendo ágar-água a $1 \%$, com abertura na tampa fixa com tecido tipo voil, sendo depositadas em cada disco cinco fêmeas adultas de T. ludeni, obtidas da criação estoque. As placas foram acondicionadas em câmara climatizada a $30 \pm 1{ }^{\circ} \mathrm{C}, 75 \% \pm 10 \%$ de umidade relativa e fotofase de 12h. Silva (2002), avaliando a biologia e exigências térmicas de $T$. ludeni em folhas de algodoeiro, cultivar CNPA ITA 90, observou que na temperatura de $30^{\circ} \mathrm{C}$ o período de ovo-adulto foi de 8,50 dias para fêmeas 7,75 dias para machos; foram efetuados experimentos individuais em delineamento experimental inteiramente casualizado, constando de quatro tratamentos (óleo e testemunha) e oito repetições.

$\mathrm{O}$ efeito dos óleos essenciais sobre o crescimento populacional de $T$. ludeni foi avaliado pela estimativa da taxa instantânea de crescimento populacional $\left(\mathrm{r}_{\mathrm{i}}\right)$, de acordo com a equação: $\mathrm{r}_{\mathrm{i}}=\ln \left(\mathrm{N}_{\mathrm{f}} / \mathrm{N}_{\mathrm{o}}\right) / \Delta \mathrm{t}$, onde: $\mathrm{N}_{\mathrm{f}}$ é o número de ácaros (ovos, imaturos e adultos) presentes em cada disco na avaliação final; $\mathrm{N}_{\mathrm{o}}$ é o número inicial das fêmeas dos ácaros transferidos para cada disco e $\Delta \mathrm{t}$ é o período de duração dos bioensaios, que foi de 10 dias (WALTHALL; STARK 1997). De acordo com a equação, se $r_{i}=0$ verifica-se o equilíbrio no crescimento populacional; se $\mathrm{r}_{\mathrm{i}}>0$, o crescimento populacional mantém-se em estado ascendente e se $\mathrm{r}_{\mathrm{i}}<0$, a população está sofrendo um declínio, que poderá levá-la à extinção, quando $\mathrm{N}_{\mathrm{f}}=0$ (STARK \& BANKS 2003). Os resultados das $\mathrm{r}_{\mathrm{i}}$ e da população final de T. ludeni foram submetidos à análise de regressão, através do programa computacional SAS version 8.02 (SAS INSTITUTE, 2001).

\section{RESULTADOS}

O óleo essencial de $C$. citriodora e M. urundeuva apresentaram efeito repelente sobre $T$. ludeni em todas as cultivares de algodão testadas. No entanto, O. basilicum apresentou efeito repelente sobre $T$. ludeni apenas para a cultivar BRS Verde (Tabela 1). O óleo de C. citriodora repeliu o ácaro em todas as cultivares, apresentando índices de repelência 0,28 a 0,56 , ou seja, não foi afetado pelas mesmas (Tabela 1)

Para o teste de $r_{i}$, a taxa instantânea de crescimento populacional, observamos que todos os valores das taxas foram positivos em todas as concentrações, indicando, segundo Stark \& Banks (2003), que a taxa da população encontra-se em estado ascendente $\left(r_{i}>0\right)$, entretanto, é possível observar que mesmo apresentando o $r_{i}$ positivo, a população 
tratada com os óleos essenciais diminuiu quando comparada com a população controle, apresentando um efeito dose dependente, em que, com o aumento das concentrações dos óleos essenciais, a população declinou. As equações de regressão foram significativas $(\mathrm{P} \leq 0,0001)$, apresentando coeficientes de determinação $\left(\mathrm{R}^{2}\right)$, variando entre 0,84 a 0,94 . De acordo com os resultados obtidos, podemos afirmar que para que se alcance um $\mathrm{r}_{\mathrm{i}}<0$, torna-se necessário o uso de concentrações de óleos essenciais maiores do que as testadas neste trabalho.

As concentrações dos óleos essenciais de eucalipto, manjericão e aroeira, em ambas as cultivares, se ajustaram ao modelo linear. Todas foram significativas, apresentando coeficientes de determinação $\left(\mathrm{R}^{2}\right)$ superiores a 0,71 . Houve interação entre concentrações e óleos essenciais nas duas cultivares tratadas, tanto para taxa instantânea de crescimento populacional (ri) (BRS Rubi: $F=17,19 ; \mathrm{P}<0,0001$ e BRS Verde: $F=34,66 ; P<0,0001$ ), quanto para a população final de T. Iudeni (BRS Rubi: $\mathrm{F}=24,98 ; \mathrm{P}<0,0001$ e BRS Verde: $\mathrm{F}=74,00 ; \mathrm{P}<0,0001)$.

Observou-se que com o aumento das concentrações dos óleos essenciais, a população final de $T$ ludeni foi se reduzindo, sendo os maiores efeitos apresentados nas maiores concentrações. O óleo de $C$. citriodora foi o que proporcionou maior redução da taxa instantânea de crescimento e da população final de T. ludeni (Figuras 1 e 2).

Tabela 1. Efeito repelente de óleos vegetais na concentração de 1,5\%, em adultos de Tetranychus ludeni, nas cultivares de algodão BRS 8H (187), BRS Rubi, BRS Safira e BRS Verde.

Table 1. Repellent effect of 1.5\% Tetranychus ludeni adult plant oils on cotton cultivars BRS 8H (187), BRS Rubi, BRS Safira and BRS Verde.

\begin{tabular}{|c|c|c|c|c|c|}
\hline \multirow[b]{2}{*}{ Cultivar } & \multirow[b]{2}{*}{ Tratamento } & \multicolumn{2}{|c|}{ Adultos atraídos (\%) } & \multirow[b]{2}{*}{$\mathrm{IR}(\mathrm{M} \pm \mathrm{DP})^{2}$} & \multirow[b]{2}{*}{ Classificação } \\
\hline & & Testemunha & Óleo $^{1}$ & & \\
\hline \multirow{3}{*}{ BRS Rubi } & Eucalyptus citriodora & 86 & $14 *$ & $0,28 \pm 0,23$ & Repelente \\
\hline & Ocimum gratissimun & 64 & $36^{*}$ & $0,72 \pm 0,30$ & Neutro \\
\hline & Myracrodruon urundeuva & 74 & $26^{*}$ & $0,52 \pm 0,23$ & Repelente \\
\hline \multirow{3}{*}{ BRS Verde } & Eucalyptus citriodora & 72 & $28 *$ & $0,56 \pm 0,17$ & Repelente \\
\hline & Ocimum gratissimun & 66 & $34 *$ & $0,68 \pm 0,11$ & Repelente \\
\hline & Myracrodruon urundeuva & 66 & $34 *$ & $0,68 \pm 0,18$ & Repelente \\
\hline
\end{tabular}
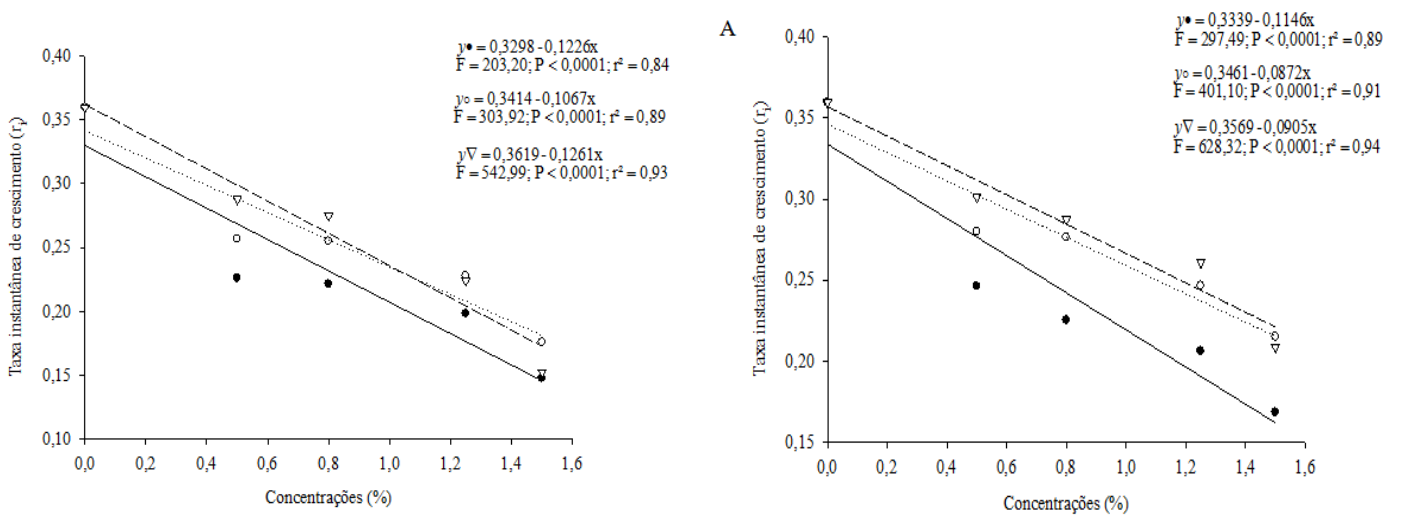

B

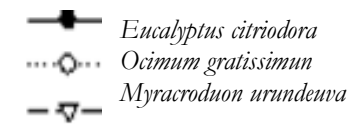

Figura 1. Taxas instantâneas de crescimento populacional ( $\mathrm{r}_{\mathrm{i}}$ ) de Tetranychus ludeni, após 10 dias, em discos de folha de algodoeiro das cultivares BRS Rubi (A) e BRS Verde (B) tratadas com diferentes concentrações de óleos essenciais.

Figure 1. Instantaneous rates of Tetranychus ludeni population growth after 10 days on cotton leaf discs of the cultivars BRS Rubi (A) and BRS Verde (B) treated with different concentrations of essential oils.

\section{DISCUSSÃO}

Segundo Olivo et al. (2008), a bioatividade do óleo de $C$. citriodora é decorrente da presença dos compostos bioativos citronelal e geraniol. Araújo Filho et al. (2018) ao analisarem a composição química do óleo essencial, confirmaram que seu composto majoritário é o citronelal com $63,9 \%$, assim como em outros trabalhos que demonstraram o mesmo resultado em relação ao composto majoritário de $C$. citriodora, como 53,1\% (HUSSEIN et al., 2017), 60,7\% (SINGH et al., 2012), 67,5\% (RIBEIRO et al., 2014), e 86,8\% (RIBEIRO et al., 2018). De acordo com Kathrina; Antônio (2004), os óleos essenciais presentes nas plantas podem causar diversos efeitos nos insetos, inclusive repelência e toxicidade, estando intimamente ligados aos seus constituintes químicos majoritários, como os compostos citronelal (87,99\%) e citronelol (8 a 20\%), encontrados em C. citriodora (MUYOBELA et al., 2016).

O potencial tóxico- repelente das plantas da família Anacardiaceae tem sido atribuído, principalmente, à presença de derivados fenólicos encontrados em suas espécies tais como taninos, saponinas, terpenos e alcaloides (PELL, 2004). Queiroz et al. (2002), relataram em pesquisas que os taninos foram os principais componentes químicos encontrados nas plantas de $M$. urundeuva. Avaliando a potencialidade inseticida de extrato de aroeira do sertão, Cavalcante et al. (2006), determinaram a presença de taninos e de alcaloides em M. urundeuva que afetaram a fertilidade de Bemisia tabaci 
(GENNADIUS, 1889), tendo reduzido a taxa de reprodução, o tempo médio de geração e a taxa intrínseca de crescimento para três gerações do inseto. Taninos são classificados como substâncias quantitativas, por serem redutores digestivos e reduzem, significativamente, o crescimento e a sobrevivência de insetos, uma vez que inativam enzimas digestivas e criam um complexo de taninos-proteínas de difícil digestão (MELLO; SILVA-FILHO, 2002).

A repelência do óleo essencial de $O$. gratissimun, foi devido, provavelmente, à ação de compostos secundários presentes nas folhas. Vieira et al. (2001) e Dambolena et al. (2010), comprovaram em seus trabalhos que o eugenol foi composto químico em maior percentual no óleo essencial da planta. No entanto, outros compostos em menores quantidades também foram encontrados como p-cimeno (22\%), o seu precursor - terpineno (15\%), e timol (17\%) (FANDOHAN et al. 2004). Estes compostos apresentam atributos farmacológicos como anticonvulsivo, anestésico, antibactericida, fungicida e acaricida ( $\mathrm{ROH}$ et al., 2013; MATUSINSKY et al., 2015). Gomes; Favero (2011), constataram ação repelente e inseticida do óleo essencial de $O$. gratissimun para Triatoma infestans (Klug, 1834), presumindo que sua ação ocorra por meio do tegumento, agindo assim, sobre o sistema nervoso.
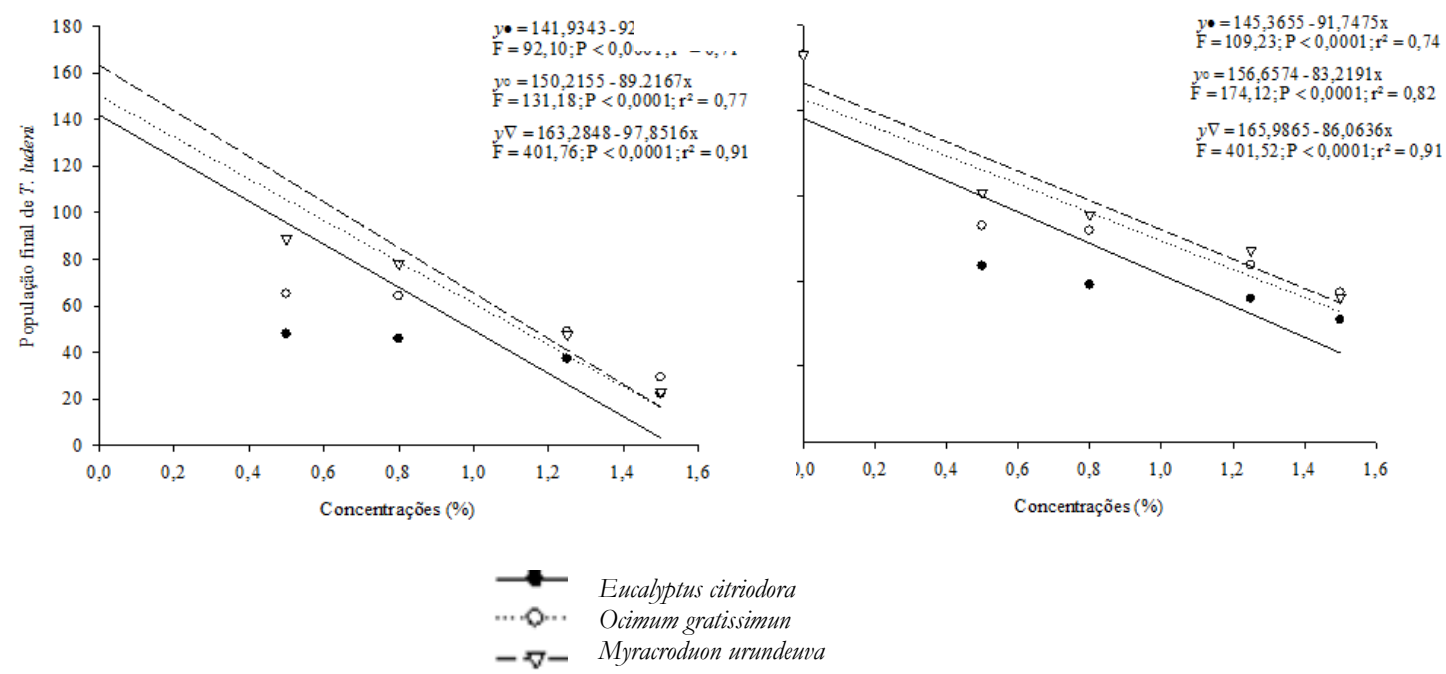

Figura 2. População final de Tetranychus ludeni, após 10 dias, em discos de folha de algodoeiro das cultivares BRS Rubi (A) e BRS Verde (B) tratadas com diferentes concentrações de óleos essenciais.

Figure 2. Final population of Tetranychus ludeni, after 10 days, on cotton leaf discs of cultivars BRS Rubi (A) and BRS Verde (B) treated with different concentrations of essential oils.

Outros estudos, visando avaliar atividades acaricidas de óleos essenciais e seus componentes químicos em ácaros fitófagos, também, foram efetuados. Efeitos acaricidas do OE de $O$. basilicum, óleo pertencente à mesma família do $O$. gratissimun, foram demonstrados sobre o ácaro rajado Tetranychus urticae Koch, este óleo apresentou alta toxicidade para fêmeas de $T$. urticae com $\mathrm{LC}_{50}$ estimado como $0,61 \mathrm{~L}^{-1}$ e $\mathrm{LC}_{90}$ como 5,9 $1 \mathrm{~L}^{-1}$, e continha linalol como a principal substância, 65,7\% (PAVELA et al., 2016). Os monoterpenos, timol e carvacrol, compostos presentes em óleos essenciais, foram repelentes para o ácaro Ixodes ricinus, apresentando em todas as concentrações testadas $90 \%$ de repelência em $I$. ricinus e também tóxicos quando testados contra Psoroptes cuniculi, ácaros de coelhos (SHANG et al., 2016; TABARI et al., 2017).

O efeito repelente significativo de algumas plantas tem sido mencionado como uma forma muito eficiente de evitar a infestação de pragas nas áreas agrícolas, e partimos da premissa que quanto maior a repelência, menor será a infestação, pelo fato de reduzir as injúrias, e consequentemente, as perdas causadas na produtividade, gerando benefícios econômicos para os agricultores.

Os produtos vegetais ou seus compostos secundários podem apresentar atividades de repelência e outros efeitos acaricidas devido a diferentes mecanismos de ação desses óleos essenciais sobre o inseto alvo: pode estar ocorrendo a neutralização dos hormônios reguladores do crescimento, inibição do desenvolvimento de ovos, ruptura do acasalamento e comunicação sexual, e inibição da formação de quitina (ROSADO-AGUILAR et al., 2017).

A taxa instantânea de crescimento populacional $\left(r_{i}\right)$, foi positiva em todas as concentrações testadas. Alguns metabólitos secundários de plantas, como alcaloides, terpenoides e compostos fenólicos, funcionam como defesa química das plantas, atuando quantitativamente, como redutores da digestibilidade, ou qualitativamente, como toxinas para os artrópodes.

A composição química dos óleos essenciais é determinada por fatores genéticos, porém, outros fatores podem acarretar alterações significativas na produção dos metabólicos secundários, como fatores abióticos como luminosidade, temperatura, pluviosidade, nutrição, época e horário de coleta, bem como técnicas de colheita e pós-colheita (BEZERRA et al., 2008). É válido ressaltar que estes fatores podem apresentar correlações entre si, não atuando isoladamente, podendo exercer influência conjunta no metabolismo secundário. Em estudos realizados com Salvia officinalis L., no qual se avaliou a influência da sazonalidade no rendimento e na composição química do seu óleo essencial, Putievsky et al. (1986) concluíram que o maior rendimento de óleo essencial foi obtido no primeiro ano de cultivo, em corte realizado no verão. 
Com relação à composição do óleo essencial, este apresentou maior teor de constituintes majoritários (tujona e cânfora) no segundo ano de cultivo, quando o corte foi realizado na primavera. Chaves et al. (2002), ao analisar o efeito da época de corte (outono, inverno, primavera e verão) na composição do óleo essencial de folhas e inflorescências de O. gratissimum demonstrou que houve interferência na composição do óleo essencial em função da variação climática, apresentando como componentes majoritários, o eugenol no verão, e o trans-cariofileno no inverno. Ao destilarem folhas de C. citriodora durante um ano, a intervalos mensais, Kapur et al. (1982) verificaram que a produção de óleo essencial foi mínima durante os meses de inverno (junho e julho), mas após esta estação, a produção aumentou gradualmente e permaneceu assim até os meses de setembro, outubro e novembro e alcançou o máximo de produção durante os meses mais quentes (dezembro a fevereiro). Observaram ainda que houve um pequeno declínio nos meses de março e abril. O teor de citronelal foi baixo em maio e junho, mas permaneceu alto nos outros meses do ano.

Lucini et al. (2010), avaliou o efeito de extrato de Capsicum baccatum (Willd) e constatou que concentrações de até $4 \%$ reduziram o crescimento populacional de T. ludeni. A taxa instantânea de crescimento populacional do ácaro vermelho do tomate, Tetranychus evansi (Baker \& Pritchard) foi zero, indicando que houve paralisação do crescimento populacional, nas concentrações de $0,62 \%, 0,72 \%, 0,93 \%$ e $1,34 \%$ para os produtos, calda sulfocálcica, NeemPro, Organic Neem e Natuneem, respectivamente (SOTO et al., 2010).

As plantas com propriedades inseticidas são fontes muito promissora de metabólitos secundários, mas que devem ser ainda muito pesquisadas em busca de compostos que possam ser utilizados no manejo integrado de pragas. Acredita-se que o estudo dos óleos essenciais possa contribuir para o desenvolvimento de produtos baseados em protótipos naturais, e desse modo essas substâncias poderiam ser utilizadas como modelos moleculares para novos inseticidas ou recomendados como compostos biorracionais nos programas de manejo de pragas. Várias substâncias provenientes dos óleos essenciais estão sendo utilizadas como alternativa aos inseticidas sintéticos, como o limoneno, utilizado nos shampoos contra pulgas, o citronelal, utilizado como repelente contra mosquitos, entre outros (GOMES; FAVERO, 2011). No entanto, para a inserção definitiva e segura dos inseticidas botânicos no mercado, estudos sobre formulações, mecanismos de ação, fitotoxidade, segurança para os consumidores e seletividade de aos inimigos naturais, dentre outros aspectos relevantes, ainda são necessários.

\section{CONCLUSÕES}

Os óleos essenciais de $C$. citriodora, O. basilicum e $M$. urundeuva exerceram repelência sobre fêmeas adultas do ácaro praga nas duas cultivares coloridas de algodões. Em relação a taxa instantânea de crescimento populacional, houve um crescimento ascendente, mas esse crescimento ocorreu a uma taxa bem menor quando comparado ao controle.

Diante dos resultados obtidos, pode-se concluir que os óleos essenciais apresentam-se como um método promissor para ser utilizado no programa de Manejo Integrado de Pragas do T. ludeni devido aos efeitos sobre a repelência e a diminuição na taxa de crescimento, além desse estudo ser de grande interesse para produtores agrícolas, uma vez que as variedades de algodão utilizadas demonstraram serem susceptíveis as atividades dos óleos essenciais, devido as substâncias bioativas, que estão contidas nos compostos de óleos essenciais, como os de C. citriodora, O. basilicum e $M$. urundeuva em relação aos compostos dos acaricidas sintéticos, como, uma mínima toxicidade para quem aplica e, para os consumidores rápida degradação e custo relativamente baixo.

\section{REFERÊNCIAS}

ADANGO, E.; ONZO, A.; HANNA, R.; ATACHI, P.; JAMES, B. Inventaire de la faune dês acariens sur Amaranthus cruentus (Amaranthaceae), Solanum macrocarpon et Solanum aethiopicum (Solanaceae) dans le Sud Bénin. International Journal of Tropical Insect Science, Wallingford, v. 26, n. 3, p. 155-165, 2006. ANDRADE, L. H.; OLIVEIRA, J. V.; BREDA, M. L.; MARQUES, E. J.; LIMA, I. M. M. Effects of botanical insecticides on the instantaneous population growth rate of Aphis gossypii Glover (Hemiptera: Aphididae) in cotton. Acta Scientiarum Agronomy, Maringá, v. 34, n. 2, p. 119-124, 2012. DOI: http://dx.doi.org/10.4025/actasciagron.v34i2.10863

ANUÁRIO BRASILEIRO DO ALGODÃO 2018. Editora Gazeta, Santa Cruz do Sul - RS, 2018. 108 p. Disponível em: http://www.editoragazeta.com.br/anuario-brasileirodo-algodao-2018/. Acesso em 25 novembro 2018.

ARAÚJO-FILHO, J. V.; RIBEIROA, W. L. C.; ANDRÉ, W. P. P.; CAVALCANTE, G. S.; GUERRA, M. C. M.; MUNIZ, C. R.; MACEDO, I. T. F.; RONDON, F. C. M.; BEVILAQUA, C. M. L.; OLIVEIRA, L. M. B. Effects of Eucalyptus citriodora essential oil and its major component, citronellal, on Haemonchus contortus isolates susceptible and resistant to synthetic anthelmintics. Industrial Crops \& Products, v. 124, p. 294-299, 2018. DOI: https://dx.doi.org/10.1016/j.indcrop.2018.07.059

BEZERRA, A. M. E.; MEDEIROS-FILHO, S.; OLIVEIRA, L. D. M.; SILVEIRA, E. R. Produção e composição química da macela em função da época de colheita. Horticultura Brasileira, Brasília, v. 26, n. 1, p. 26-29, 2008. DOI: http://dx.doi.org/10.1590/S010205362008000100005

CAVALCANTE, G. M.; MOREIRA, A. F. C.; VASCONCELOS, S. D. Potencialidade inseticida de extratos aquosos de essências florestais sobre mosca branca. Pesquisa Agropecuária Brasileira, Brasília, v. 41 n. $1, \quad$ p. $9-14, \quad 2006$. DOI: http://dx.doi.org/10.1590/S0100-204X2006000100002

CECÍlLIO, A. B.; OLIVEIRA, P. DE C.; CALDAS, S.; CAMPANA, P. R. V.; FRANCISCO, F. L.; DUARTE, M. G. R.; DE ALMEIDA, V. L. Antiviral activity of Myracrodruon urundeuva against rotavirus. Revista Brasileira de Farmacognosia, São Paulo, v. 26, n. 2, p. 197-202, 2016. DOI: http://dx.doi.org/10.1016/j.bjp.2015.10.005

CHAVES, F. C. M.; MING, L. C.; CARVALHO, E. A. V.; FERNANDES, D. M.; MARQUES, M. O. M.; MEIRELES, M. A. M. Produção de biomassa, rendimento de óleo essencial e teor de eugenol em alfavaca-cravo, em função da adubação orgânica e sazonalidade. Horticultura Brasileira, Brasília, v. 20, n. 2, julho, 2002. CLEMENTE, M. A.; MONTEIRO, C. M. O.; SCORALIK, M. G.; GOMES, F. T.; PRATA, M. C. A.; DAEMON, E. Acaricidal activity of the essential oils from Eucalyptus 
citriodora and Cymbopogon nardus on larvae of Amblyomma cajennense (acari: ixodidae) and Anocentor nitens (Acari: Ixodidae). Parasitology Research, Berlin, v. 107, n. 4 , p. 987-992, 2010. DOI: https://dx.doi.org/10.1007/s00436-010-1965-0

CONAB_COMPANHIA NACIONAL DE ABASTECIMENTO. Produto algodão em pluma. Disponível em: <http//www.conab.gov.br.> Acesso em 30 de maio 2018.

DAMBOLENA, J. S.; ZUNINO, M. P.; LÓPEZ, A. G.; RUBINSTEIN, H. R.; ZYGADLO, J. A.; MWANGI, J. W.; Kariuki, S. T. Essential oils composition of Ocimum basilicum L. and Ocimum gratissimum L. from Kenya and their inhibitory effects on growth and fumonisin production by Fusarium verticillioides. Innovative Food Science \& Emerging Technologies, Amsterdam, v. $11 \mathrm{n}$. 2, p. $\quad 410-414, \quad 2010$. DOI: https://dx.doi.org/10.1016/j.ifset.2009.08.005

DE MENDONÇA ALBUQUERQUE, R. J.; LEAL, L. K. A. M.; BANDEIRA, M. A.; VIANA, G. S. B.; RODRIGUES, L. V.; Chalcones From Myracrodruon Urundeuva Are Efficacious In Guinea Pig Ovalbumin-Induced Allergic Conjunctivitis. Revista Brasileira de Farmacognosia, v. 21, p. 953-962, 2011.

EMBRAPA ALGODÃO_EMPRESA BRASILEIRA DE PESQUISA AGROPECUÁRIA. Cultivo do Algodão herbáceo na agricultura familiar. Cultivares. 2. ed. 2006. (Sistema de Produção, 1) Disponível em: $<$ http://sistemasdeproducao.cnptia.embrapa.br/FontesHT ML/Algodao/Algodao Agricultura Familiar_2ed/cultivares.html> Acesso em: 30/05/2018.

ESTEVES FILHO, A. B.; OLIVEIRA, J. V.; TORRES, J. B.; GONDIM JR. M. G. C. Biologia comparada e comportamento de Tetranychus urticae Koch (Acari: Tetranychidae) e Phytoseiulus macropilis (Banks) (Acari: Phytoseiidae) em Algodoeiro Bollgard ${ }^{\mathrm{TM}}$ e Isolinha nãoTransgênica. Neotropical Entomology, v.39, n.3, p.338344 , 2010.

http://www.scielo.br/pdf/ne/v39n3/v39n3a05.pdf

FANDOHAN, P.; GBENOU, J. D.; GNONLONFIN, B.; HELL, K.; MARASAS, W. F. O.; WINGFIELD, M. J. Effect of essential oils on the growth of Fusarium verticillioides and Fumonisin contamination in corn. Journal of Agricultural and Food Chemistry, v. 52, n. 22, p. 6824-6829, 2004.

FINNEY, D.J. Probit analysis. 3rd ed. London: Cambridge University Press, 1971. 333p.

FLECHTMANN, C. H. W. Ácaros de importância agrícola. 6. ed. São Paulo: Nobel, 1985. 189 p.

GALLO, D.; NAKANO, O.; SILVEREIRA NETO, S.; CARVALHO, R. P. L.; BAPTISTA, G. C.; BERTI FILHO, E.; PARRA, J. R. P.; ZUCCHI, R. A.; ALVES, S. B.; VENDRAMIN, J. D.; MARCHINI, L. C.; LOPES, J. R. S.; OMOTO, C. Entomologia Agrícola. São Paulo: FEALQ, v. 10, 2002. 920 p.

GOMES, S. P.; FAVERO, S. Avaliação de óleos essenciais de plantas aromáticas com atividade inseticida em Triatoma infestans (Klug, 1834) (Hemiptera: Reduviidae). Acta Scientiarum. Health Sciences, Maringá, v. 33, n. 2, p. 147-151, 2011. DOI: http://dx.doi.org/10.4025/actascihealthsci.v33i2.9531

GOMES, V. T. L.; CHAVES, T. P.; ALENCAR, L. C. B.; DANTAS, I. C.; DE MEDEIROS, A. C. D.; FELISMINO,
D. C. Antimicrobial activity of natural products from Myracrodruon urundeuva Allemão (Aroeira-do-sertão). Revista Cubana de Plantas Medicinales, v. 18, n. 4, p. 529-533, 2013.

HASEGAWA, T.; TAKATA, F.; NIIYAMA, T.; OHTA, M. Bioactive monoterpene glycosides conjugated with gallic acid from the leaves of Eucalyptus globulus. Phytochemistry, v. 69, n. 3, p. 747-753, 2008. DOI: https://dx.doi.org/10.1016/j.phytochem.2007.08.030

HUSSEIN, H. S.; SALEM, M. Z. M.; SOLIMAN, A. M. Repellent, attractive, and insecticidal effects of essential oils from Schinus terebinthifolius fruits and Corymbia citriodora leaves on two whitefly species, Bemisia tabaci, and Trialeurodes ricini. Scientia Horticulturae, Amsterdam, v. 216, p. 111-119, 2017. DOI: https://dx.doi.org/10.1016/j.scienta.2017.01.004

KATHRINA, G. A.; ANTONIO, L. O. J. Controle biológico de insectos mediante extratos botânicos. In: CARBALL, M.; GUAHARAY, F. (Ed.). Control biológico de plagas agrícolas. Managua: CATIE, 2004. p. 137-160.

KAIMAL, S. G.; N. RAMANI. Feeding biology of Tetranychus ludeni Zacher (Acari: Tetranychidae) on velvet bean. Systematic and Applied Acarology, London, v. 16, n. 3, p. 228-234, 2011. DOI: https://dx.doi.org/10.11158/saa.16.3.7

KAPUR, K. K; VASHIST, V. N.; ATAL, C. K. Variability and utilization studies on Eucalyptus citriodora Hook. Grown in India. In: ATAL, C.K; KAPUR, B.M. (eds). Cultivation and utilization of aromatic plants. JammuTawi: Regional Laboratory Council of Scientific and Industrial Research, 1982. p. 603-606.

KHATER, H.; HENDAWY, N.; GOVINDARAJAN, M.; MURUGAN, K.; BENELLI, G. Photosensitizers in the fight against ticks: safranin as a novel photodynamic fluorescent acaricide to control the camel tick Hyalomma dromedarii (Ixodidae). Parasitology Research, Berlin, v. 115 , n. 10 , p. 3747-3758, 2016. DOI: https://dx.doi.org/10.1007/s00436-016-5136-9

KOUSIK, C. S.; SHEPARD, B. M.; HASSELL, R.; LEVI, A.; SIMMONS, A. M. Potential sources of resistance to broad mites Polyphagotarsonemus latus in watermelon germplasm. HortScience, Alexandria, v. 42, n. 1, p. 15391544 , 2007.

DOI: https://dx.doi.org/10.21273/HORTSCI.42.7.1539

KHOURY, M.; STIEN, D.; EPARVIER, V.; OUAINI, N.; EL BEYROUTHY, M. Report on the medicinal use of eleven lamiaceae species in Lebanon and rationalization of their antimicrobial potential by examination of the chemical composition and antimicrobial activity of their essential oils. Evidence-Based Complementary and Alternative Medicine, v. 16, p. 1-18, 2016. DOI: http://dx.doi.org/10.1155/2016/2547169

KUMRAL, N. A.; COBANOGLU, S.; YALCIN, C. Acaricidal, repellent and oviposition deterrent activities of Datura stramonium L. against adult Tetranychus urticae (Koch). Journal of Pest Science, v. 83, n. 2, p. 173-180, 2010. DOI: https://dx.doi.org/10.1007/s10340-0090284-7

LUCINI, T.; SCABENI, C.; DEDORDI, C.; HIROSE, E.; SHIOMI, H. F. Efeito de extrato aquoso de Capsicum baccatumna mortalidade e oviposição de Tetranychus ludeni (Acari: Tetranychidae). Scientia Agraria, Curitiba, 
v. 11, n. 4, p. 355-358, 2010. DOI: http://dx.doi.org/10.5380/rsa.v11i4.18272

LUPI, E.; HATZ, C.; SCHLAGENHAUF, P. The efficacy of repellents against Aedes, Anopheles, Culex and Ixodes spp.-A literature review. Travel Medicine and Infectious Disease, v. 11, p. 374-411, 2013. DOI: https://dx.doi.org/10.1016/j.tmaid.2013.10.005

MATUSINSKY, P.; ZOUHAR, M.; PAVELA, R.; NOVY, P. Antifungal effect of five essential oils against important pathogenic fungi of cereals. Industrial Crops and Products, v. 67, p. 208-215, 2015. DOI: https://dx.doi.org/10.1016/j.indcrop.2015.01.022

MAMADALIEVA, N.Z.; AKRAMOV, D.K.; OVIDI, E.; TIEZZI, A.; NAHAR, L.; AZIMOVA, S.S.; SARKER, S.D. Aromatic medicinal plants of the lamiaceae family from uzbekistan: ethnopharmacology, essential oils composition, and biological activities. Medicines, v. 4, n. 1, $\quad$ p. $\quad 8, \quad 2017 . \quad$ DOI: https://dx.doi.org/10.3390/medicines4010008

MELLO, M. O.; SILVA-FILHO, M. C. Plant-insect interactions: an evolutionary arms race between two distinct defense mechanisms. Brazilian Journal of Plant Physiology, Londrina, v. 14, n. 2, p. 71-81, 2002. DOI: http://dx.doi.org/10.1590/S1677-04202002000200001

MOSCARDINI, V. F.; GONTIJO, P. C.; MICHAUD, J. P.; CARVALHO, G. A. Sublethal effects of chlorantraniliprole and thiamethoxam seed treatments when Lysiphlebus testaceipes feed on sunflower extrafloral néctar. Biocontrol, Dordrecht, v. 59, n. 5, p. 503- 511, $2014 . \quad$ DOI: https://dx.doi.org/10.1007/s10526-014-9588-5

MUYOBELA, J.; YOBE-NKUNIKA, P. O.; TEMBOMWASE, E. In vitro acaricidal activity of Bobgunnia madagascariensis Desv. against Amblyomma variegatum (Fabricius) (Acari: Ixodidae). Tropical Animal Health and Production, Edinburgh, v. 48, n. 3, p. 625631, 2016. DOI: https://dx.doi.org/10.1007/s11250-0161009-6

NAPOLEÃO, T. H.; PONTUAL, E. V.; DE ALBUQUERQUE LIMA, T.; DE LIMA SANTOS, N. D.; SÁ, R. A.; COELHO, L. C.; DO AMARAL FERRAZ NAVARRO, D. M.; PAIVA, P. M. Effect of Myracrodruon urundeuva leaf lectin on survival and digestive enzymes of Aedes aegypti larvae. Parasitology Research, Berlin, v. 110, n. 2, p. 609-616, 2012. DOI: https://dx.doi.org/10.1007/s00436-011-2529-7

NASCIMENTO, S. S.; ARAUJO, A. A. S.; BRITO, R. G.; SERAFINI, M. R.; MENEZES, P. P.; DESANTANA, J. M. Cyclodextrin-complexed Ocimum basilicum leaves essential oil increases fos protein expression in the central nervous system and produce an antihyperalgesic effect in animal models for fibromyalgia. Internacional Journal of Molecular Science, v. 16, n. 1, p. 547-563, 2015. DOI: https://dx.doi.org/10.3390/ijms16010547

OLIVO, C. J. CARVALHO, N. M.; SILVA, J. H. S.; VOGEL, F. F.; MASSARIOL, P.; MEINERZ, G.; AGNOLIN, C.; MOREL, A. F.; VIAU, L. V. Óleo de citronela no controle do carrapato de bovinos. Ciência Rural, Santa Maria, v. 38 , n. 2, p. 406-410, 2008. DOI: https://doi.org/10.1590/S0103-84782008000200018

PAVELA, R.; STEPANYCHEVA, E.; SHCHENIKOVA, A.; CHERMENSKAYA, T.; PETROVA, M. Essential oils as prospective fumigants against Tetranychus urticae Koch.
Industrial Crops and Products, v. 94, p. 755-761, 2016. DOI: http://dx.doi.org/10.1016/j.indcrop.2016.09.050

PELL, S. K. (2004). Molecular systematics of the cashew family (anacardiaceae). Dissertation in Doctor of Philosophy Biological Sciences, Faculty of the Louisiana State University. May.

PUTIEVSKY, E.; RAVID, U.; DUDAI, N. The influence of season and harvest frequency on essential oil and herbal yields from a pure clone of sage (Salvia officinalis L.), grown under cultivated conditions. Journal of Natural Products, Cincinnati, v. 49, n. 2, p. 326-329, 1986. DOI: https://dx.doi.org/10.1021/np50044a023

QUEIROZ, C. R. A. A.; MORAIS, S. A. L.; NASCIMENTO, E. A. Caracterização dos taninos da aroeira-preta (Myracrodruon urundeuva). Revista Árvore, Viçosa, v. 26, p. 485-492, 2002. DOI: http://dx.doi.org/10.1590/S0100-67622002000400011

RIBEIRO, J. C.; RIBEIRO, W. L. C.; CAMURÇAVASCONCELOS, A. L. F.; MACEDO, I. T. F.; SANTOS, J. M. L.; PAULA, H. C. B.; ARAÚJO-FILHO, J. V.; MAGALHÃES, R. D.; BEVILAQUA, C. M. L. Efficacy of free and nanoencapsulated Eucalyptus citriodora essential oils on sheep gastrointestinal nematodes and toxicity for mice. Veterinary Parasitology, Amsterdam, v. 204, p. 243-248, 2014. DOI: https://dx.doi.org/10.1016/j.vetpar.2014.05.026

RIBEIRO, A. V.; FARIAS, E. S.; SANTOS, A. A.; FILOMENO, C. A.; SANTOS, I. B.; BARBOSA, L. C. A.; PICANÇO, M. C. Selection of an essential oil from Corymbia and Eucalyptus plants against Ascia monuste and its selectivity to two non-target organisms. Crop Protection, Guildford, v. 110, p. 207-213, 2018. DOI: https://dx.doi.org/10.1016/j.cropro.2017.08.014

ROH, A. S.; LEE, B. H.; PARK, C. G. Acaricidal and repellent effects of myrtacean essential oils and their major constituents against Tetranychus urticae (Tetranychidae). Journal of Asia-Pacific Entomology, v. 16, n. 3, p. 245249, 2013.2 DOI: https://dx.doi.org/10.1016/j.aspen.2013.03.001

ROSADO-AGUILAR, J. A.; ARJONA-CAMBRANES, K.; TORRES-ACOSTA, J. F. J.; RODRÍGUEZ-VIVAS, R. I.; BOLIO-GONZÁLEZ, M. E.; ORTEGA-PACHECO, A.; AGUILAR-CABALLERO, A. J. Plant products and secondary metabolites with acaricide activity against ticks. Veterinary Parasitology, Amsterdam, v. 238, p. 66-76, 2017. DOI:

http://dx.doi.org/doi:10.1016/j.vetpar.2017.03.023

SAS INSTITUTE. SAS/STAT User`s guide, version 8.2, TS level 2MO. Cary: SAS Institute. Inc., 2001.

SANTOS, H. G. Sistema brasileiro de classificação de solos. 3. ed. Brasília, Embrapa, 2013. 306 p.

SHANG, X.; WANG, YU.; ZHOU, X.; GUO, X.; DONG, S.; WANG, D.; ZHANG, J.; PAN, H.; ZHANG, Y.; MIAO, $\mathrm{X}$. Acaricidal activity of oregano oil and its major component, carvacrol, thymol and p-cymene against Psoroptes cuniculi in vitro and in vivo. Veterinary Parasitology, Amsterdam, v. 226, p. 93- 96, 2016. DOI: https://dx.doi.org/10.1016/j.vetpar.2016.07.001

SINGH, H. P.; KAUR, S.; NEGI, K.; KUMARI, S.; SAINI, V.; KOHLI, R. K.; BATISH, D. R. Assessment of in vitro antioxidant activity of essential oil of Eucalyptus citriodora (lemon-scented eucalypt; Myrtaceae) and its major constituents. Food Science Technology, London, v. 
48, n. 2, p. 237-241, 2012. DOI: https://dx.doi.org/10.1016/j.lwt.2012.03.019

SOTO, A.; VENZON, M.; OLIVEIRA, R.; OLIVEIRA, H. G.;

PALLINI, A. Alternative control of Tetranychus evansi Baker \& Pritchard (Acari: Tetranychidae) on tomato plants grown in greenhouses. Neotropical Entomology, Londrina, v. 39, n. 4, p. 638-44, 2010. DOI: http://dx.doi.org/10.1590/S1519-566X2010000400027

TABARI, M. A.; YOUSSEFI, M. R.; MAGGI, F.; BENELLI, G. Toxic and repellent activity of selected monoterpenoids (thymol, carvacrol and linalool) against the castor bean tick, Ixodes ricinus (Acari: Ixodidae). Veterinary Parasitology, Amsterdam, v. 245, p. 86-91, 2017. DOI: http://dx.doi.org/10.1016/j.vetpar.2017.08.012

TOLBA, H.; MOGHRANI, H.; BENELMOUFFOK, A.; KELLOU, D.; MAACHI, R. Essential oil of Algerian Eucalyptus citriodora: Chemical composition, antifungal activity. Journal de Mycologie Médicale, Paris, v. 25, p. 128-133, 2015.

DOI: https://doi.org/10.1016/j.mycmed.2015.10.009

VIEIRA, R. F.; GRAYER, R. J.; PATON, A.; SIMON, J. E. Genetic diversity of Ocimum gratissimum L. based on volatile oil constituents, flavonoids and RAPD markers. Biochemical Systematics and Ecology, Oxford, v. 29, n. 3, p. 287-304, 2001. DOI: https://dx.doi.org/10.1016/S0305-1978(00)00062-4

WALTHALL, W. K.; STARK, J. D. A comparison of acute mortality and population growth rate as endpoints of toxicological effect. Ecotoxicology and Environmental Safety, v.37, p.45-52, 1997.

ZHANG, Z.-Q. Taxonomy of Tetranychus ludeni (Acari: Tetranychidae) in New Zealand and its ecology on Sechium edule. New Zealand Entomologist, Nelson, v. 25, p. 27-34, 2002. https://dx.doi.org/10.1080/00779962.2002.9722091 